\title{
EFFECT OF FE CONTENT ON THE MICROSTRUCTURE AND MECHANICAL PROPERTIES OF Ti-Al-Mo-V-Cr-Fe ALLOYS
}

\begin{abstract}
To investigate the effect of $\mathrm{Fe}$ content on the correlation between the microstructure and mechanical properties in near-b titanium alloys, the Ti-5Al-5Mo-5V-1Cr-xFe alloy system has been characterized in this study. As the Fe content increased, the number of nucleation sites and the volume fraction of the $\alpha$ phase decreased. We observed a significant difference in the shape and size of the $\alpha$ phase in the matrix before and after Fe addition. In addition, these morphological deformations were accompanied by a change in the shape of the $\alpha$ phase, which became increasingly discontinuous, and changed into globular-type $\alpha$ phase in the matrix. These phenomena affected the microstructure and mechanical properties of Ti alloys. Specimen \#2 exhibited a high ultimate tensile strength $(1071 \mathrm{MPa})$, which decreased with further addition of $\mathrm{Fe}$.
\end{abstract}

Keywords: Ti alloy, Near- $\beta$ titanium alloy, Aircraft application, Structural material, Landing gear

\section{Introduction}

Ti-based alloys are used in the aerospace, automotive, and medical industries because of their attractive properties $[1,2]$. The unusual combination of light weight, high specific strength, toughness, corrosion, and oxidation resistance makes Ti-based alloys attractive for aerospace applications [3-5]. Because of these outstanding properties, Ti-based alloys are in used as materials in gas turbine engine parts, fuselage, wings, compressor discs and blades, and spacers and landing gear as structural components in the aerospace industry. When Ti-based alloys are used in an actual product, the $\alpha+\beta$ phase Ti-6Al-4V (Ti-64) alloys are dominant. However, the share of $\beta$ or near- $\beta$ phase Ti-based alloys in aerospace applications has grown substantially in the last decade because of it has high strength and greater hardening ability than the $\alpha+\beta$ phase Ti-based alloys. For example, Boeing 777 is the first commercial aircraft with near- $\beta$ phase Ti-based alloy, Ti-10V$2 \mathrm{Fe}-3 \mathrm{Al}$ (Ti-1023). The number of components comprising this alloy has outnumbered components with the $\alpha+\beta$ Ti-based alloy because of its improved fatigue performance and weldability [6].

The use of $\beta$ or near- $\beta$ phase Ti-based alloys is growing in the aerospace industry, where historically the industry has benefited from the unusual combination of desirable properties. Although the high cost of these alloys has been a factor in their rate of use, most of the applications have been driven because of the increased performance [7].

In particular, near- $\beta$ phase Ti-based alloys, such as Ti-1023,
(Ti-55531), are suitable for use in aircraft structural components because of their light weight, strong mechanical properties, good processability and thermal treatability [8-10].

The recently developed alloy $\mathrm{Ti}-5 \mathrm{Al}-5 \mathrm{Mo}-5 \mathrm{~V}-1 \mathrm{Cr}-1 \mathrm{Fe}$ (Ti-55511) had been identified as a potential material for the truck beam component of the landing gear of Boeing 787 and Airbus A-380. Aircraft structural applications such as landing gear need good mechanical properties, especially ultimate tensile strength (UTS) $\left(\sigma_{\max } 900-1400 \mathrm{MPa}\right)$ and fracture toughness $\left(\mathrm{K}_{\mathrm{IC}}>60 \mathrm{MPa}\right)$, to be considered for use in components for safe take-off and landing of aircrafts [11-13].

The microstructure of near- $\beta$ Ti alloys consists of the $\alpha$ and $\beta$ phases where the amount and shape of the $\alpha$ phase controls the mechanical properties to a large extent. Moreover, heat treatment is used to modify the microstructure of this category of alloys. Aging is the final and inevitable processing step that is used to improve the mechanical properties. Strengthening by precipitation of the $\alpha$ phase is then achieved during aging heat treatments [13]. The concept of substituting Fe, which is inexpensive and gives the alloys high performance, in part or completely for the expensive $\mathrm{V}$ or Mo, which are commonly used in many commercial alloys, has been discussed for a long time.

In this study, to investigate the effect of the Fe content on the relationship between the microstructure and the mechanical properties, four near- $\beta$ Ti alloys, Ti-5Al-5Mo-5V-1Cr-xFe $(\mathrm{x}=0,1,3,5 \mathrm{wt} . \%)$ were produced

* DEPARTMENT OF MATERIALS SCIENCE AND ENGINEERING, PUSAN NATIONAL UNIVERSITY, 2 , BUSANDAEHAK-RO 63BEON-GIL, GEUMJEONG-GU, BUSAN 46241, REPUBLIC OF KOREA ** MATERIAL ANALYSIS LABORATORY, DAE-IL CORPORATION, ULSAN, REPUBLIC OF KOREA

*** SOCIAL ENTERPRISE, PUSAN NATIONAL UNIVERSITY, BUSAN, REPUBLIC OF KOREA 


\section{Experimental}

The purity of each element used in the ingot was more than $3 \mathrm{~N}$-grade. Ingots with a nominal composition of Ti-5Al$5 \mathrm{Mo}-5 \mathrm{~V}-1 \mathrm{Cr}-\mathrm{xFe}(\mathrm{x}=0,1,3,5 \mathrm{wt} . \%)$, as shown in Table 1 , were prepared by vacuum arc remelting (VAR) mixtures in an Ar gas atmosphere. The ingots were remelted 10 times with 5 flippings for each ingot until they were completely homogenized. Then, characterization of the $\beta$-transition temperature was performed by differential thermal analysis (DTA).

TABLE 1

The chemical composition of Ti-5Al-5Mo-5V-1Cr-xFe alloy (wt. \%)

\begin{tabular}{|c|c|c|c|c|c|c|}
\hline \hline Specimen & Ti & Al & Mo & V & Cr & Fe \\
\hline$\# 1$ & Bal. & $5 \pm 0.3$ & $5 \pm 0.2$ & $5 \pm 0.2$ & $1 \pm 0.2$ & 0 \\
\hline$\# 2$ & Bal. & $5 \pm 0.3$ & $5 \pm 0.2$ & $5 \pm 0.2$ & $1 \pm 0.2$ & $1 \pm 0.1$ \\
\hline$\# 3$ & Bal. & $5 \pm 0.3$ & $5 \pm 0.2$ & $5 \pm 0.2$ & $1 \pm 0.2$ & $3 \pm 0.1$ \\
\hline$\# 4$ & Bal. & $5 \pm 0.3$ & $5 \pm 0.2$ & $5 \pm 0.2$ & $1 \pm 0.2$ & $5 \pm 0.1$ \\
\hline
\end{tabular}

The heat treatment process is shown in Fig. 1. Each sample was recrystallized above the $\beta$-transition temperature at $950^{\circ} \mathrm{C}$ for $2 \mathrm{~h}$, and then quenched in water. Then, each sample was annealed at $700^{\circ} \mathrm{C}$ for $2 \mathrm{~h}$ and air cooled (A.C.) to room temperature (R.T.). All the heat treatments were performed in Ar gas atmosphere.

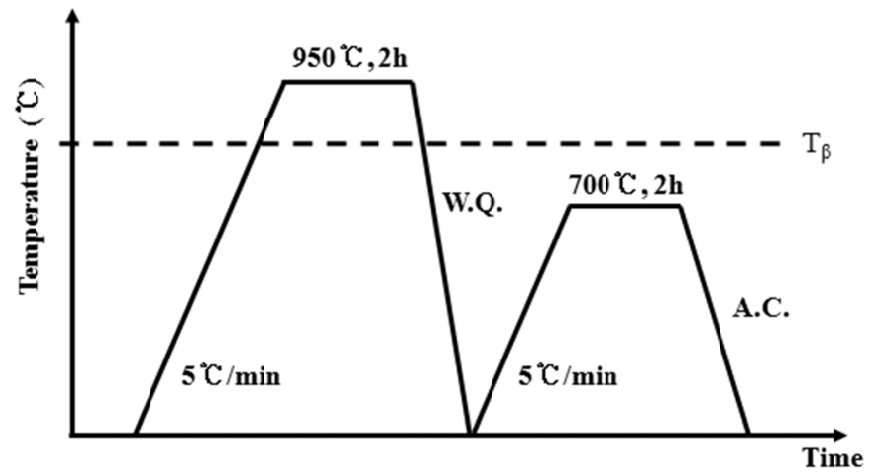

Fig. 1. Heat treatment processing route for $\beta$ annealed microstructure in near- $\beta$ titanium alloys

The structure of each alloy was determined by X-ray diffraction (XRD, Rigaku Ultima IV, Japan) using $\mathrm{CuK} \alpha$ radiation $(\lambda=0.154 \mathrm{~nm})$ over a $2 \theta$ range of $30-90^{\circ}$. The microstructure of each alloy was observed by field emission scanning electron microscopy (FE-SEM, TESCAN MIRA 3, Czech Republic) in the secondary electron (SE) and back-scattered electrons (BSE) mode. The specimens was etched by Kroll's reagent and observed in SE mode FE-SEM. The mechanical properties of each alloy were evaluated by tensile test using ASTM E8. The tensile properties for each specimen were tested by a universal testing machine with a strain rate for $1.5 \times 10^{-4} \mathrm{~s}^{-1}$ at R.T. and the fracture surface was observed using a FE-SEM.

\section{Results and discussion}

Fig. 2 and Table 2 show the XRD patterns of all the specimens and the volume fraction change of the $\alpha$ and $\beta$ phase, respectively. The volume fraction change in each phase was estimated by the X-ray intensity of each phase. The addition of Fe as a $\beta$-phase stabilizer caused an increase or decrease in the volume fraction of the $\alpha$ and $\beta$ phases by $17 \%$, as shown in Table 2 .

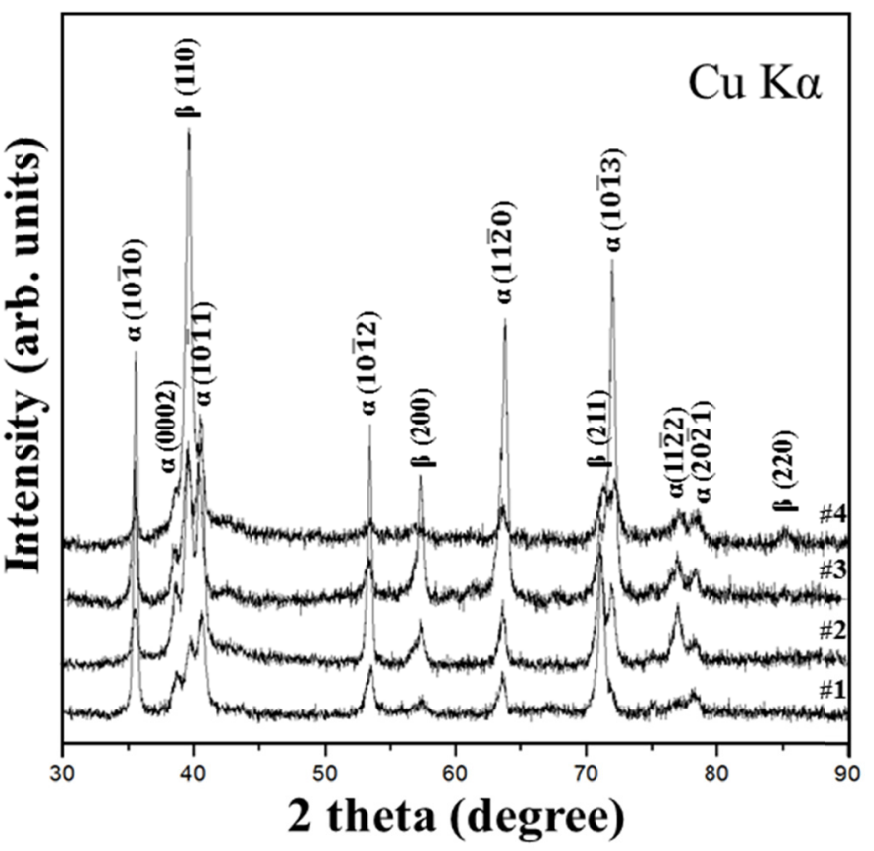

Fig. 2. XRD results of each alloy

TABLE 2

Volume fraction and mechanical properties of each alloy

\begin{tabular}{|c|c|c|c|c|c|}
\hline \hline Specimen & $\boldsymbol{V}_{\boldsymbol{\alpha}} \mathbf{( \% )}$ & $\boldsymbol{V}_{\boldsymbol{\beta}} \mathbf{( \% )}$ & $\mathbf{U T S}(\mathbf{M P a})$ & $\boldsymbol{E} \mathbf{( G P a )}$ & $\boldsymbol{\varepsilon}$ \\
\hline$\# 1$ & $51 \pm 3$ & $49 \pm 3$ & 975 & 114 & 0.85 \\
\hline$\# 2$ & $49 \pm 2$ & $51 \pm 2$ & 1071 & 110 & 0.97 \\
\hline$\# 3$ & $39 \pm 3$ & $61 \pm 3$ & 957 & 105 & 0.91 \\
\hline$\# 4$ & $34 \pm 1$ & $66 \pm 1$ & 874 & 101 & 0.86 \\
\hline
\end{tabular}

Fig. 3 shows the FE-SEM images of the microstructure of each specimen in the SE and BSE modes. The microstructure consist of primary $\alpha$, secondary $\alpha, \beta$, and the retained $\beta$ phases. Although the grain boundary of $\alpha$ is observed in Fig. 3(a-b), no grain boundary of $\alpha$ is observed in Fig. 3(c-d). A short and discontinuous $\alpha$ phase was observed around the grain boundary of the $\alpha$ phase in Fig. 3(b). To observe these $\alpha$ phases in detail, specimens were etched and a thicker and shorter $\alpha$ phase was observed in Fig. 3(b) BSE than SE mode. As Fe is added, the nucleation site of the $\alpha$ phase reduced and the shape of the $\alpha$ phase became increasingly discontinuous and short. The shape of the $\alpha$ phase changed into a plate and globular type from the lath- and acicular-type Fig. 3(c-d). The white circle in Fig. 3(b) indicates the grown secondary $\alpha$ and $\beta$ phase region [14]. To analyze the shape of the $\alpha$ phase in the white circle, these specimens were etched by Kroll's reagent. As a result, the shape of the grown $\alpha$ phase exhibits a lath-type structure with a thickness for less 

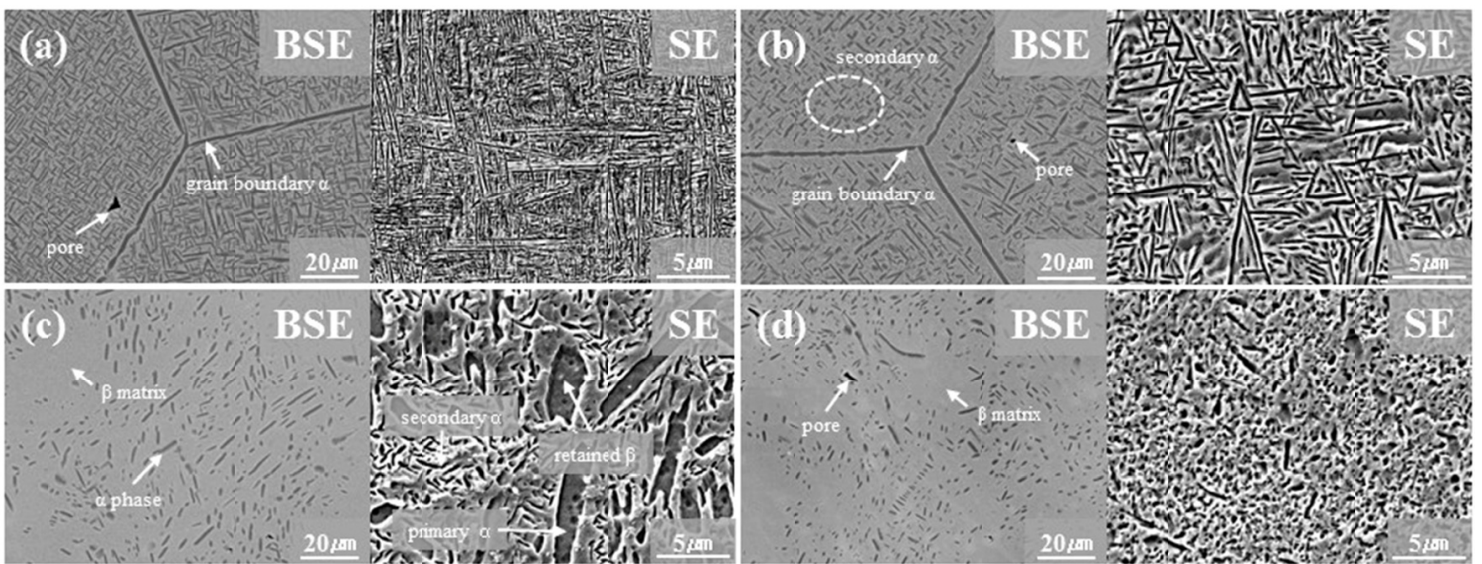

Fig. 3. FE-SEM images of each alloy: (a) \#1, (b) \#2, (c) \#3, and (d) \#4

than $1 \mu \mathrm{m}$ in Fig. 3(b) SE. As shown in Fig. 3(c) SE, not fully transformed into a single $\alpha$ phase and this means that the retained $\beta$ phase exists as a plate-type structure in the $\alpha$ phase. It is considered that recrystallization of the $\beta$ phase occurs during aging [15]. Globular-type $\alpha$ phase is observed in Fig. 3(d) SE, which is uniformly distributed in the $\beta$ matrix.

Fig. 4 shows the stress-strain behavior of each specimen. In the tensile test, specimen \#2 exhibits the highest UTS (1071 MPa) compared to other specimens. The UTS decreases upon further addition of Fe. This results from the change in the shape and volume fraction of the $\alpha$ phase, which is a key factor determining the mechanical properties in near- $\beta$ Ti alloys [16]. It is considered that as the shape of the $\alpha$ phases change into globular type from the lath type, the volume fraction of the $\alpha$ phase decreased. These phenomena are related to the change in the microstructure, as shown Fig. 3. Regardless of the UTS value, the Young's modulus decreased from 114 to $101 \mathrm{GPa}$ as the Fe content increased.

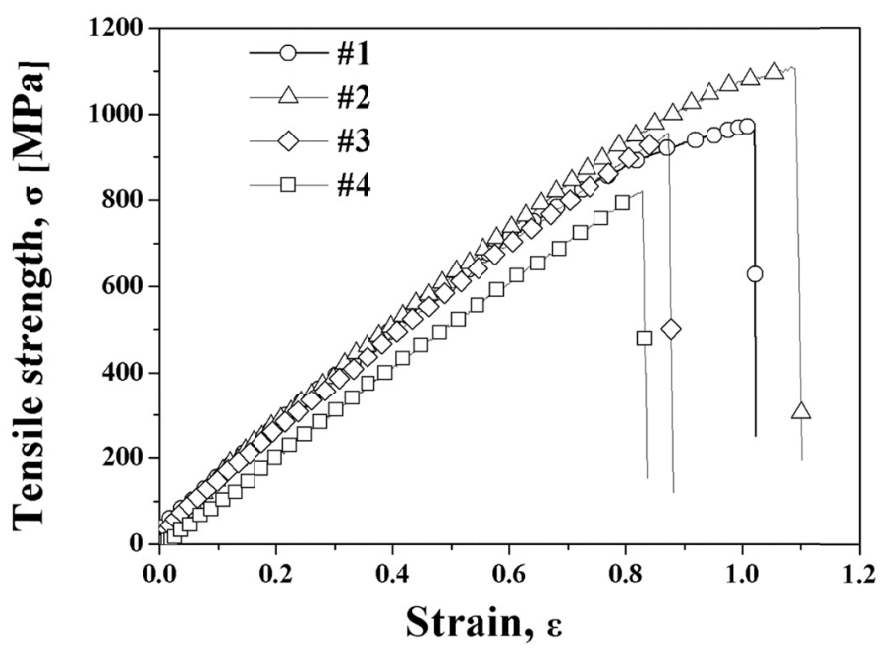

Fig. 4. Stress-strain curves of each alloy

Fig. 5 shows the low and high magnification FE-SEM images of the fracture surface by tensile testing. As shown in Fig. 5(a-b-c), it mainly consists of a mixed brittle and ductile fracture surface with a shape remarkably similar to that of the $\alpha$ phase in the microstructure. Brittle fracture was considered as the failure that occurred along the $\alpha$ phase. The low magnification image in Fig. 5(a) shows a mixed mode type of fracture. The cleavage faceted surface of the $\alpha$ phase and the dimpled surface of the $\beta$ phase around these $\alpha$ phases were observed in area a-1, and an intergranular fracture surface was observed in area a-2. Comparing Fig. 5(b) and Fig. 5(a), a faceted fracture surface of the $\alpha$ phase, which is thicker and more elongated than the dimpled surface of the $\beta$ phase around the $\alpha$ phases, was observed in area b-1. In Fig. 5(b) area b-2, shallow and numerous small dimples that are situated within the larger dimple were observed. As shown in Fig. 5(c), a cleavage fracture surface was observed at the plate type $\alpha$ phases, but mainly deep dimpled fracture surfaces that occurred at the $\beta$ phases were observed. Furthermore, a deep dimple was observed in Fig. 5(c) area c-2, which implies that a completely ductile fracture mode occurred in this area. Fig. 5(d) shows that a predominantly ductile fracture occurred and a river pattern was observed at low magnification. Ductile fracture occurred along the river pattern as shown in Fig. 5(d) areas d-1 and d-2.

\section{Conclusions}

In this study, the microstructure and mechanical properties of near- $\beta$ titanium alloys, Ti-5Al-5Mo-5V-1Cr-xFe with different Fe content fabricated using the VAR process have been studied. The results are summarized as follows:

1. The addition of $\mathrm{Fe}$ as a $\beta$ phase stabilizer caused the volume fraction of the $\alpha$ phase increased and the volume fraction of the $\beta$ phase decreased.

2. The addition of $\mathrm{Fe}$ as a $\beta$ phase stabilizer caused the shape of the $\alpha$ phase to change into the plate- and globular-type from the lath- and acicular-type.

3. In case of the tensile test, the \#2 specimen exhibits the highest UTS among all the specimens, but it decreased upon further addition of Fe. Regardless of the UTS value, the Young's modulus decreased from 114 to $101 \mathrm{GPa}$ as the Fe content increased.

4. The fracture surface consists of mixed brittle and ductile mode in specimens \#1, 2, and 3, but a predominantly ductile fracture occurred in specimen \#4 along with river patterns. 

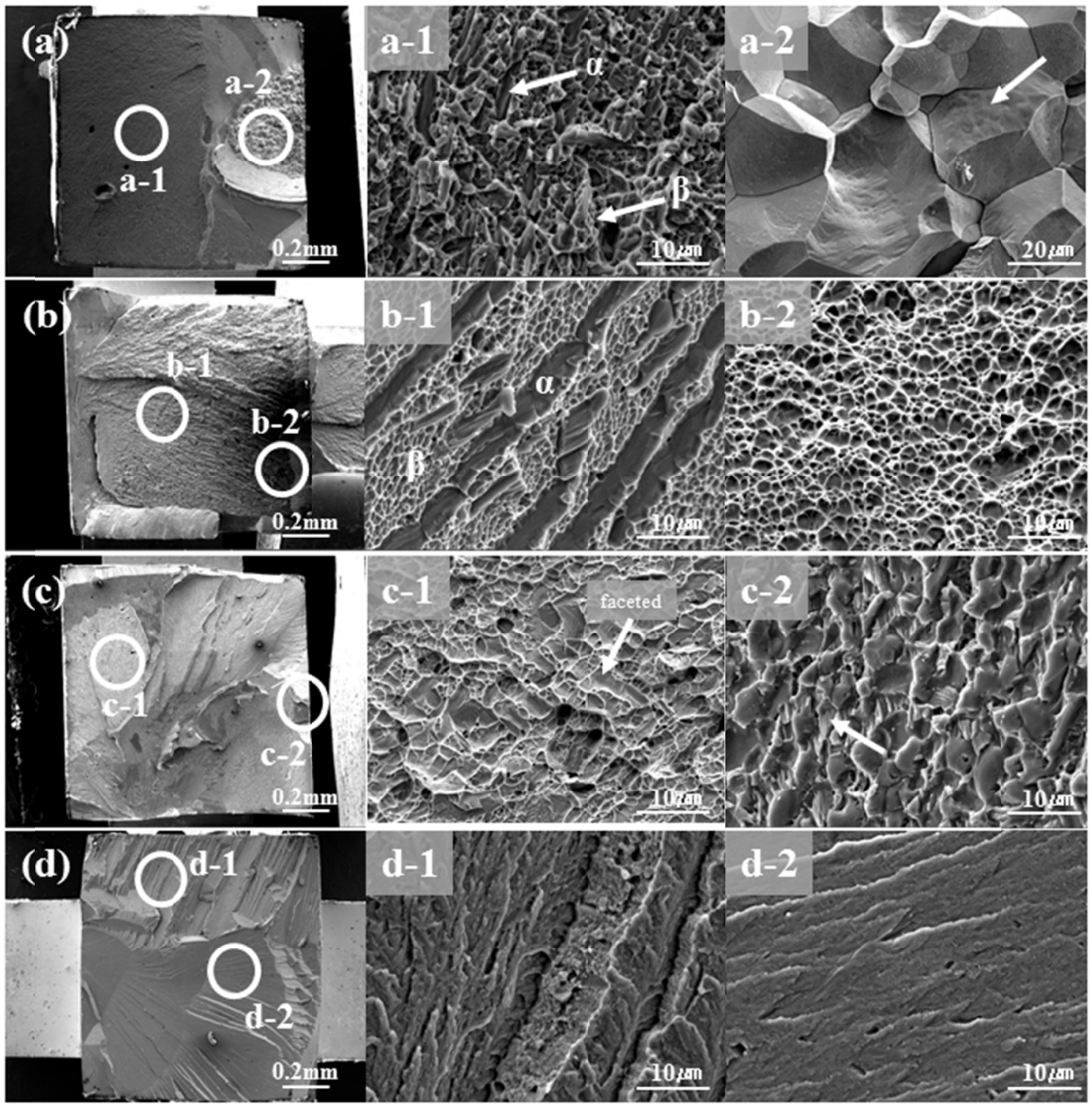

Fig. 5. Low and high magnification FE-SEM images of fracture surface by tensile test: (a) \#1, (b) \#2, (c) \#3, and (d) \#4

Acknowledgments

This work was supported by BK21PLUS, Social Enterprise Specialist Development Group.

\section{REFERENCES}

[1] J.N. Hwang, M.J. LEE, H.J. Kim, I.H. Oh, K.A. Lee, Journal of Korean Powder Metallurgy Institute 19, 5, 348 (2012).

[2] Y.M. Kim, Y.B. Song, S.H. Lee, Y.S. Kwon, Journal of Korean Powder Metallurgy Institute 21, 4, 277 (2014).

[3] C. Leyens, M. Peters, Titanium and titanium allloys, Wiley Online Library (2005).

[4] J.C. Williams, E.A. Starke Kr, Acta Materialia 51, 5775 (2001).

[5] F. Froes, D. Eylon, International Materials Reviews 35, 162 (1990).

[6] R. Boyer, Materials Science and Engineering A 213, 103 (1996).
[7] R. Boyer, R. Briggs, Journal of Materials Engineering and Performance 14, 681 (2005).

[8] M. Jackson, N. Jones, D. Dye, R. Dashwood, Materials Science and Engineering A 501, 248 (2009).

[9] P.J. Arrazola, A. Garay, L.M. Iriarte, M. Armendia, S. Marya, F. Le Maitre, Journal of Material processing technology 209, 2223 (2009).

[10] F. Warchomicka, C. Poletti, M. Stockinger, Materials Science and Engineering A 528, 207 (2011).

[11] N. Richards, J. Barnby, Materials Science and Engineering A 26, 221 (1976).

[12] V.N. Moiseev, Metal Science and Heat Treatment 40, 11 (1998.

[13] V.N. Moiseev, Metal Science and Heat Treatment 42, 81 (2000).

[14] M. Ahmed, D.G. Savvakin, O.M. Ivasishin, E.V. Pereloma, Materials Science and Engineering A 576, 167 (2013).

[15] J.K. Fan, H.C. Kou, M.J. Lai, B. Tang, H. Chang, J.S. Li, Mater. Sci. Eng. A 584, 121 (2013).

[16] S. Shekhar, R. Sarkar, Materials \& Design. 66, 596 (2015). 